\title{
DIOPHANTINE APPROXIMATION BY \\ PRIME NUMBERS, III
}

\author{
By R. C. VAUGHAN
}

[Received 25 November 1974]

\section{Introduction}

We continue our study of diophantine approximation by prime numbers. The problem that we study in this paper arises naturally from the subject matter of [13], but our approach will differ from that of either [13] or [14]. However, before passing to a description of the contents of this paper we make some remarks concerning [14]. Let $\lambda_{1}, \lambda_{2}, \ldots, \lambda_{s}$ be $s$ non-zero real numbers, not all of the same sign and not all in rational ratio. Let $\eta$ be any real number. As in [14] we define $\mathscr{D}(k)$ to be the least $s$ for which there is a positive real number $\sigma$ such that the inequality

$$
\left|\eta+\sum_{j=1}^{s} \lambda_{j} p_{j}{ }^{k}\right|<\left(\max p_{j}\right)^{-\sigma}
$$

has infinitely many solutions in prime numbers $p_{j}$. The number $D(k)$ is the corresponding value for $s$ if we insist only that the variables are natural numbers. The major interest in the results of [14] lies in the bounds for the number of variables rather than that on the right of (1.1) we have a power of the maximum value of the variables. For this reason bounds are given only when $k \geqslant 4$ and $k \geqslant 5$ respectively, that is, only when the bounds for the number of variables are better than those known previously even when there is only a fixed $\varepsilon$ on the right of (1.1).

By combining the methods of [13] and [14] with Theorem 4 of Hua [7] we can easily show that $\mathscr{D}(2) \leqslant 5$ and $\mathscr{D}(3) \leqslant 9$. As far as $D$ is concerned, when $k=2$ a great deal is known and we have nothing new to add. When $k=4$ the method of [14] will give $D(4) \leqslant 14$, and when $k=3$ the method can be combined with that of Davenport and Roth [4] to give $D(3) \leqslant 8$. In each of these, of course, the bound for the number of variables is not new. What is new is that the right of (1.1) contains a power of the maximum of the variables.

In this paper we are concerned with the following question, posed by Halberstam in conversation. Suppose that $\lambda_{1} / \lambda_{2}$ is negative and irrational. Then can one use sieve methods to show that there exists a positive integer $k$ such that the numbers of the form $\lambda_{1} p+\lambda_{2} P_{k}$, with $p$ a 
prime number and $P_{k}$ a natural number having at most $k$ prime factors, are dense on the real line? We answer this question in the affirmative and show that 4 is a permissible value for $k$.

We use results of Richert [10] and a new idea of Chen, who has recently shown [1] (for a shorter proof see Ross [11]) that every large even integer is of the form $p+P_{2}$. We also require two analogues of the BombieriVinogradov mean value theorem (see Chapter 24 of Davenport [2]) and these form the bulk of our work. Here the exponent $\frac{1}{2}$ which appears in the Bombieri-Vinogradov theorem has to be replaced by $\frac{1}{4}$ and is the cause of our being unable to do better than $k=4$. It has been found that the most satisfactory approach is to adapt the arguments of [15].

Our main theorem is as follows.

TheOREM. There is a positive number $\tau$ such that if $\lambda_{0}, \lambda_{1}$, and $\lambda_{2}$ are real numbers with $\lambda_{1} / \lambda_{2}<0$ and $\lambda_{1} / \lambda_{2}$ irrational, then there are infinitely many prime numbers $p$ for which there exists a square free natural number $P_{4}$, having at most four prime factors, such that

$$
\left|\lambda_{0}+\lambda_{1} p+\lambda_{2} P_{4}\right|<p^{-\tau} \text {. }
$$

It clearly suffices to prove the theorem with

$$
\lambda_{2}=-1, \quad \lambda_{1}>0,
$$

which we assume henceforward.

\section{Notation and assumed results}

Throughout $p$ is a prime number, $a, g, k, m, n, q, r$ are natural numbers, $j, b, h$ are integers, $t, \alpha, \lambda, \sigma$ are real numbers, $u, v, x, y, T, X, \eta, \xi$ are real numbers greater than or equal to $1, z$ is a real number with

$$
0<z<\min \left(\lambda_{1}, 1 / \lambda_{1}\right)
$$

$\delta$ is a real number satisfying $0<\delta \leqslant \frac{1}{4}$, and $\varepsilon$ is a sufficiently small positive real number in terms of $\delta$. Implied constants in the $O$ and $\ll$ notations depend at most on $\delta, \varepsilon, \lambda_{0}$, and $\lambda_{1}$. We write $\|\alpha\|=\min _{n}|\alpha-n|$ and if $\alpha+\frac{1}{2}$ is not an integer we use $\llbracket \alpha \rrbracket$ to denote the integer nearest to $\alpha$. We further define $\mathscr{L}=\log y$, and given an arbitrary function $f, B(\sigma, f)$ is used to denote the formal expression

$$
\sum_{n \leqslant \eta} \sum_{\chi \bmod r r}^{*} \int_{-T}^{T}|f(\sigma+i t, \chi)| \frac{d t}{1+|t|} .
$$

Here $\Sigma_{x}^{*}$ denotes summation over all the primitive characters modulo $n r$. We reserve $d, \varphi, \omega, \Lambda$, and $\Omega$ for respectively the divisor function, Euler's function, the number of different prime divisors, von Mangoldt's function, 
and the total number of prime divisors. As usual $\psi(x)=\Sigma_{n \leqslant x} \Lambda(n)$, $\pi(x)=\Sigma_{p \leqslant x} 1$, and $L(s, \chi)$, where $s=\sigma+i$, denotes the Dirichlet $L$ function formed from the character $\chi$. The letters $\mathscr{A}, \mathscr{D}, \mathscr{M}$, and $\mathscr{N}$ denote sets of integers and $|\mathscr{A}|$ is the cardinality of $\mathscr{A}$. We further use $\mathscr{A}_{r}$ to denote $\{h: h \in \mathscr{A}, r \mid h\}$.

For our proof of (1.2) we require the following results from multiplicative number theory.

Lemma 2.1. Suppose that $T \geqslant 2$. Then

and

$$
\sum_{\chi \bmod n}^{*} \int_{-T}^{T}\left|L\left(\frac{1}{2}+i t, \chi\right)\right|^{4} \frac{d t}{1+|t|} \ll \varphi(n) \log ^{5} n T
$$

$$
\sum_{\chi \bmod n}^{*} \int_{-T}^{T}\left|L^{\prime}\left(\frac{1}{2}+i t, \chi\right)\right|^{4} \frac{d t}{1+|t|} \ll \varphi(n) \log ^{9} n T .
$$

Proof. Theorem 10.1 of Montgomery [8] states that

The inequality

$$
\Sigma_{\chi}^{*} \int_{-T}^{T}\left|L\left(\frac{1}{2}+i t, \chi\right)\right|^{4} d t \ll \varphi(n) T \log ^{4} n T .
$$

$$
\sum_{\chi}^{*} \int_{-T}^{T}\left|L^{\prime}\left(\frac{1}{2}+i t, \chi\right)\right|^{4} d t \ll \varphi(n) T \log ^{8} n T
$$

can be shown in the same way as Corollary 10.2 of Montgomery [8]. The lemma now follows by partial summation.

Lemma 2.2. Let $T \geqslant 2$ and

$$
U(s, \chi)=\sum_{m} c_{m} \chi(m) m^{-i t},
$$

where the summation is over any set of positive integers $m$ for which $\Sigma_{m}\left|c_{m}\right|^{2} m$ exists. Then

$$
B\left(0, U^{2}\right) \ll \sum_{m}\left|c_{m}\right|^{2}\left(m+\eta^{2} r \log T\right) .
$$

Proof. By Theorem 1 of Davenport and Halberstam [3], with the $x_{j}$ the set of points $a / n r$ with $1 \leqslant a \leqslant n r,(a, n r)=1$, and $n \leqslant \eta$, we have

$$
\sum_{n \leqslant \eta} \sum_{\substack{a=1 \\(a, n r)=1}}^{n r}\left|\sum_{m=M+1}^{M+N} c_{m} m^{-i t} e(a m / n r)\right|^{2} \ll\left(N+\eta^{2} r\right) \sum_{m=M+1}^{M+N}\left|c_{m}\right|^{2} .
$$

We then complete the proof of the lemma by using the method of Theorem 3 of Gallagher [6] combined with (5) of Gallagher [5], and performing a partial integration. 
LEMMA 2.3. Suppose that

$$
\begin{gathered}
0<\alpha \leqslant 1, \\
1<\alpha u \leqslant 4 \\
0<\lambda<1 \\
\sum_{n \leqslant x^{\alpha}} 3^{\omega(n)}\left|\sum_{b \in \mathscr{N}_{n}} 1-x n^{-1}\right|<x / \log ^{2} x \\
P(v)=\prod_{p<v} p
\end{gathered}
$$

and

$$
W(\mathscr{A}, u, \lambda)=\sum_{\substack{b \in \mathscr{A} \\
\left(b, P\left(x^{\alpha / 4}\right)\right)=1}}\left(1-\sum_{\substack{\alpha / 4 \leqslant p<x^{1 / u} \\
p \mid b}} \lambda\left(1-\frac{u \log p}{\log x}\right)\right)+\sum_{\substack { p \geqslant x^{\alpha / 4} \\
\begin{subarray}{c}{b \in \mathcal{A} \\
p^{2} \mid b{ p \geqslant x ^ { \alpha / 4 } \\
\begin{subarray} { c } { b \in \mathcal { A } \\
p ^ { 2 } | b } }\end{subarray}} 1,
$$

where $\Sigma^{\prime}$ means that those $b$ which have a repeated prime factor are not counted in the summation. Then

$$
W(\mathscr{A}, u, \lambda) \geqslant \frac{2 x}{\alpha \log x}\left(\log 3-\lambda \alpha \int_{u}^{4 / \alpha} \frac{t-u}{t \alpha-1} \frac{d t}{t}+O\left((\log x)^{-1 / 15}\right)\right) .
$$

This, $\dagger$ apart from a few trivial modifications, is a special case of Theorem 1 of Richert [10].

LEMMA 2.4. Suppose that (2.3) and (2.6) hold. Then

$$
S\left(\mathscr{A}, x^{\alpha}\right)=\sum_{\substack{b \in \mathcal{\infty} \\\left(b, P\left(x^{\alpha}\right)\right)=1}} 1 \leqslant \frac{2 x}{\alpha \log x}\left(1+O\left((\log x)^{-1 / 14}\right)\right) .
$$

This follows from Theorem B of Richert [10].

Lemma 2.5 (Pólya-Vinogradov). Let $\chi$ be a non-principal character modulo $n$. Then

$$
\sum_{m \leqslant u} \chi(m) \ll n^{1 / 2} \log n
$$

For a proof of this see, for instance, p. 146 of Prachar [9].

LeMmA 2.6. Let $f(s)=\sum_{n=1}^{\infty} a_{n} n^{-8}(\sigma>1)$ where $a_{n} \ll \log 2 n$. Then for every natural number $N$ and every $\theta>1$,

$$
\sum_{n \leqslant N} a_{n}=\frac{1}{2 \pi i} \int_{\theta-i T}^{\theta+i T} f(s) \frac{\left(N+\frac{1}{2}\right)^{s}}{s} d s+O\left(\frac{\left(N+\frac{1}{2}\right)^{\theta}}{T}\left(\frac{1}{(\theta-1)^{2}}+\log ^{2} N\right)\right) .
$$

This is a special case of Lemma 3.12 of Titchmarsh [12].

$\dagger$ Note added in proof. Proofs of Lemmas 2.3 and 2.4 can be found in the recent book of Halberstam and Richert [6a]. 


\section{An analogue of the Bombieri-Vinogradov theorem}

Let

and

$$
\begin{gathered}
\mathscr{D}=\left\{\llbracket \lambda_{0}+\lambda_{1} p \rrbracket: p \leqslant y,\left\|\lambda_{0}+\lambda_{1} p\right\| \leqslant \frac{1}{2} z\right\}, \\
R_{r}=\left|\mathscr{D}_{r}\right|-x r^{-1}, \quad x=z \pi(y),
\end{gathered}
$$

$$
\psi(v, r, h, m)=\sum_{\substack{n \leqslant v \\ h n \equiv m(\bmod r)}} \Lambda(n) .
$$

In (3.1), if $\llbracket \lambda_{0}+\lambda_{1} p_{1} \rrbracket=\llbracket \lambda_{0}+\lambda_{1} p_{2} \rrbracket$ with $p_{1} \neq p_{2}$, then the elements $\llbracket \lambda_{0}+\lambda_{1} p_{j} \rrbracket$ are considered as distinct. In order to sieve the set $\mathscr{D}$ we require information concerning $R_{r}$, which is provided by the following theorem.

Theorem 3.1. Suppose that $0<\delta \leqslant \frac{1}{4}$ and $\left|\lambda_{1}-a / q\right| \leqslant q^{-2}$ with $(a, q)=1$ and $q \geqslant q_{0}\left(\delta, \lambda_{0}, \lambda_{1}\right)$. Let $y=q^{2 /(1+\delta)}$. Then

$$
\sum_{r \leqslant y^{1 / 4-\delta}}\left|R_{r}\right| \ll y^{1-\delta / 4}
$$

The proof of (3.4) rests on the following lemma.

\section{LeMma 3.1. Let}

and

$$
\Psi(u, v, r, h)=\sum_{\substack{m, u u \\(m, r)=1}}\left(\psi(v, r, h, m)-\frac{\psi(v)}{\varphi(r)}\right)
$$

$$
\Psi(r)=\max |\Psi(u, v, r, h)|,
$$

where the maximum is taken over all $h, u, v$ with $(h, r)=1, u \leqslant q\left(2+\left|\lambda_{0}\right|\right)$, and $v \leqslant y$ respectively. Then

$$
\sum_{m \leqslant y^{1 / 4-\delta}} \Psi(m q) \ll y^{1-(2 \delta / 7)}
$$

We defer the proof of this lemma until the next section and proceed with the deduction of (3.4) from (3.7).

Write $\lambda_{0}=\left(b+\theta_{0}\right) / q$ and $\lambda_{1}=a / q+\theta_{1} q^{-2}$ where $\left|\theta_{0}\right|,\left|\theta_{1}\right| \leqslant 1$, and suppose that $p \leqslant y$. Then

$$
\lambda_{\theta}+\lambda_{1} p=\left(b+a p+\theta_{0}\right) / q+\theta_{1} p q^{-2} .
$$

Now write $b+a p=j+h q$ with $-\frac{1}{2} q<j \leqslant \frac{1}{2} q$. Then

$$
\lambda_{0}+\lambda_{1} p-h=\left(j+\theta_{0}\right) / q+\theta_{1} p q^{-2} .
$$

Thus $-\frac{1}{2} z \leqslant \lambda_{0}+\lambda_{1} p-h \leqslant \frac{1}{2} z$ if and only if

$$
-\frac{1}{2} z q \leqslant j+\theta_{0}+\theta_{1} p / q \leqslant \frac{1}{2} z q .
$$

Consider the inequality

$$
|j| \leqslant \frac{1}{2} z q .
$$


Clearly all but $O(y / q)=O\left(q^{1-\delta}\right)$ possible values of $j$ which satisfy (3.8) also satisfy (3.9) and vice versa. Moreover, if $-\frac{1}{2} z \leqslant \lambda_{0}+\lambda_{1} p-h \leqslant \frac{1}{2} z$, we must have $h=\llbracket \lambda_{0}+\lambda_{1} p \rrbracket$ and in this case $r \mid \llbracket \lambda_{0}+\lambda_{1} p \rrbracket$ if and only if $r \mid h$, that is, if and only if $b+a p \equiv j(\bmod q r)$. If $j$ is exceptional then $h$ either is $\llbracket \lambda_{0}+\lambda_{1} p \rrbracket$ or differs from $\llbracket \lambda_{0}+\lambda_{1} p \rrbracket$ by 1 . In the latter case, $r \mid \llbracket \lambda_{0}+\lambda_{1} p \rrbracket$ implies that either $b+a p \equiv j+q(\bmod q r)$ or $b+a p \equiv j-q$ $(\bmod q r)$. Thus, by $(3.1)$,

$$
\left|\mathscr{D}_{r}\right|=\sum_{|j| \leqslant \ddagger z q} \sum_{\substack{p \leqslant y \\ b+a p=j(\bmod q r)}} 1+O\left(q^{1-\delta}\left(\frac{y(a, r)}{q r}+1\right)\right) .
$$

Therefore, by (3.2),

$$
\sum_{r \leqslant v^{1 / 4-\delta}}\left|R_{r}\right| \ll y d(a) \mathscr{L} q^{-\delta}+\sum_{r \leqslant y^{1 / 4}-\delta}\left|z r^{-1} \pi(y)-\sum_{|j| \leqslant 1 z q} \sum_{\substack{p \leqslant y \\ b+a p=j(\bmod q r)}} 1\right| .
$$

Let

Then

$$
g=(a, r) \text {. }
$$

$$
\begin{aligned}
& \sum_{|j| \leqslant \downarrow z q} \sum_{\substack{p \leqslant y \\
b+a p \equiv j(\bmod q r)}} 1=\sum_{\substack{h \\
|b+h| \leqslant \operatorname{tad} q a}} \sum_{a p=h(\bmod q r)} 1 \\
& =\sum_{\substack{j \\
\left|b g^{-1}+j\right| \leqslant z q / 2 g}} \sum_{\substack{p \leqslant y, p \nmid q r / g \\
a p / g \equiv j(\bmod q r / g)}} 1+O(\mathscr{L}) .
\end{aligned}
$$

Hence, by (3.10) and (3.11),

$$
\begin{aligned}
& \sum_{r \leqslant y^{1 / 4-8}}\left|R_{r}\right| \\
& \ll y d(a) \mathscr{L} q^{-\delta}+\sum_{n \mid a} \sum_{\substack{m \leqslant 1 / 1 /-\delta / n \\
(m, a / n)=1}}\left(\left|\sum_{\substack{j,|b n-1,+j| \leqslant z q / 2 n \\
(j, m q)=1}}\left(\frac{\pi(y)}{\varphi(m q)}-\sum_{\substack{p \leqslant y \\
a p / n=j(\bmod m q)}} 1\right)\right|\right. \\
& \left.+\pi(y)\left|\frac{z}{m n}-\sum_{\substack{j,|b n-4, j| \leqslant z q / 2 n \\
(j, m q)=1}} \frac{1}{\varphi(m q)}\right|\right) .
\end{aligned}
$$

It is easily verified that

$$
\sum_{\substack{u<j \leqslant 0 \\(j, r)=1}} 1=\frac{\varphi(r)}{r}(v-u)+O(d(r)),
$$

and we recall that $\lambda_{0}=\left(b+\theta_{0}\right) / q$, so that $|b| \leqslant q\left(1+\left|\lambda_{0}\right|\right)$. Hence

$$
\begin{aligned}
\sum_{r \leqslant v^{1 / 4-\delta}} \mid & R_{r} \mid \\
& \ll y d(a) \mathscr{L}_{q^{-\delta}}+d(a) \sum_{m \leqslant y^{1 / 4-\delta}-\delta} \max _{\substack{x \leqslant q\left(2+1 \lambda_{0}\right) \\
(h, m q)=1}}\left|\sum_{\substack{j \leqslant x \\
(j, m q)=1}}\left(\frac{\pi(y)}{\varphi(m q)}-\sum_{\substack{p \leqslant y \\
h p=j(\bmod m q)}} 1\right)\right| \\
& \ll y d(a) \mathscr{L}_{q^{-\delta}}+d(a) \sum_{m \leqslant y^{1 / 4-\delta}} \Psi(m q),
\end{aligned}
$$


where we have used the inequality

$$
\sum_{2 \leqslant n \leqslant y} c_{n} \ll \max _{v \leqslant y}\left|\sum_{n \leqslant v} c_{n} \log n\right| .
$$

The theorem now follows from (3.13) and (3.7).

\section{Proof of Lemma 3.1}

Suppose that $(h, m q)=1, m q \leqslant \xi \leqslant y, \quad u \leqslant q\left(2+\left|\lambda_{0}\right|\right)$, and $v \leqslant y$. Consider the expression $\Psi(u, v, m q, h)$ given by (3.5). We use the inequality

$$
\left|\psi(v)-\sum_{\substack{n \leqslant v \\(n, m q)=1}} \Lambda(n)\right| \ll \mathscr{L}^{2}
$$

to replace $\psi(v)$ by $\sum_{n \leqslant v,(n, m q)=1} \Lambda(n)$. We then write the resulting expression in terms of characters modulo $m q$. Next we replace each character $\chi$ modulo $m q$ by the primitive character $\chi^{*}$ that induces it, making use of the inequality

$$
\left|\psi(v, \chi)-\psi\left(v, \chi^{*}\right)\right| \ll \mathscr{L}^{2}
$$

and Lemma 2.5. Furthermore, we note that

$$
\sum_{n \leqslant u} \chi(n) \ll d(m q) \max _{k \leqslant u}\left|\sum_{n \leqslant k} \chi^{*}(n)\right| .
$$

Hence, by (3.6),

Thus

$$
\Psi(m q) \ll \frac{d(m q)}{\varphi(m q)} \sum_{\chi \neq \chi \bmod m q} \max _{\substack{u \leqslant q\left(2+\left|\lambda_{0}\right|\right) \\ v \leqslant y}}\left|\sum_{k \leqslant u} \chi^{*}(k) \psi\left(v, \chi^{*}\right)\right|+m^{1 / 2} q^{1 / 2} \mathscr{L}^{3} .
$$

$$
\begin{aligned}
& \sum_{m \leqslant \xi} \Psi(m q) \ll \xi^{3 / 2} q^{1 / 2} \mathscr{L}^{3}+y^{\varepsilon} q^{-1} \sum_{r \mid q} \sum_{r^{-1}<n \leqslant \xi} n^{-1} \sum_{\chi_{\bmod n r}} \max _{\substack{u\left(2+\mid \lambda_{0}\right) \\
v \leqslant y}}\left|\sum_{k \leqslant u} \chi(k) \psi(v, \chi)\right| \\
& \ll \xi^{3 / 2} q^{1 / 2} \mathscr{L}^{3}+y^{e} q^{-1} \sum_{r \mid q}\left(\xi^{-1} \Sigma_{1}(\xi)+\int_{1}^{\xi} \Sigma_{1}(\eta) \eta^{-2} d \eta\right),
\end{aligned}
$$

where

$$
\Sigma_{1}(\eta)=\sum_{r-1<n \leqslant \eta} \sum_{\chi \bmod n r}^{*} \max _{\substack{u \leqslant q\left(2+\left|\lambda_{0}\right|\right) \\ v \leqslant y}}\left|\sum_{k \leqslant u} \chi(k) \psi(v, \chi)\right|
$$

By Hölder's inequality,

where

$$
\Sigma_{1}(\eta) \leqslant \Sigma_{2}^{1 / 4} \Sigma_{3}^{3 / 4}
$$

and

$$
\Sigma_{2}=\sum_{r^{-1}<n \leqslant \eta} \sum_{\chi \bmod n}^{*} \max _{u \leqslant q\left(2+\left|\lambda_{0}\right|\right)}\left|\sum_{k \leqslant u} \chi(k)\right|^{4}
$$

$$
\Sigma_{3}=\sum_{r^{-1}<n \leqslant \eta} \sum_{\chi \bmod n r}^{*} \max _{v \leqslant y}|\psi(v, \chi)|^{4 / 3}
$$


Let

and

$$
\begin{gathered}
T=y^{100} \\
\theta=1+\mathscr{L}^{-1}
\end{gathered}
$$

Then, by Lemma 2.6,

$$
u_{0}=\frac{1}{2}+\max _{m \leqslant u} m
$$

$$
\sum_{k \leqslant u} \chi(k)=\frac{1}{2 \pi i} \int_{\theta-i T}^{\theta+i T} L(s, \chi) \frac{u_{0}^{s}}{s} d s+O(1) .
$$

We note that $L(s, \chi)$ is regular for $\sigma>0$, and

Thus

$$
L(s, \chi) \ll m^{1 / 2} q^{1 / 2}(1+|t|)^{1 / 2} \quad\left(\sigma \geqslant \frac{1}{2}\right) .
$$

$$
\sum_{k \leqslant u} \chi(k)=\frac{1}{2 \pi i} \int_{1 / 2-i T}^{1 / 2+i T} L(s, \chi) \frac{u_{0}^{s}}{s} d s+O(1) .
$$

Hence, by Hölder's inequality and (2.1),

$$
\begin{aligned}
\sum_{\chi_{\bmod n r}}^{*} \max _{u \leqslant q\left(2+\left|\lambda_{0}\right|\right)}\left|\sum_{k \leqslant u} \chi(k)\right|^{4} & \ll q^{2} \mathscr{L}^{3} B\left(\frac{1}{2}, L^{4}\right)+\varphi(n r) \\
& \ll q^{2} \varphi(n r) \mathscr{L}^{8} .
\end{aligned}
$$

Therefore, by (4.4),

We next treat $\Sigma_{3}$. Let

$$
\begin{gathered}
\Sigma_{2} \ll \eta^{2} q^{2} r \mathscr{L}^{8} . \\
v_{0}=\frac{1}{2}+\max _{m \leqslant v} m, \\
F(s, \chi)=\sum_{n \leqslant \eta r^{1 / 3}} \Lambda(n) \chi(n) n^{-8}
\end{gathered}
$$

and

$$
G(s, \chi)=\sum_{n \leqslant y^{1 / 2}} \mu(n) \chi(n) n^{-s} .
$$

Then, by Lemma 2.6, for a non-principal character $\chi$, we have

$$
\begin{aligned}
2 \pi i \psi(v, \chi)= & \int_{\theta-i T}^{\theta+i T}\left(\frac{L^{\prime}}{L}+F\right)(L G-1)(s, \chi) \frac{v_{0}^{s}}{s} d s \\
& +\int_{1 / 2-i T}^{1 / 2+i T}\left(F-L^{\prime} G-L F G\right)(s, \chi) \frac{v_{0}^{s}}{s} d s+O(1) .
\end{aligned}
$$

Hence, by (4.5) and Hölder's inequality, applied several times,

$\Sigma_{3} \ll \mathscr{L}^{1 / 3} y^{4 / 3} B\left(\theta,\left|\left(L^{\prime} / L+F\right)(L G-1)\right|^{4 / 3}\right)$

$$
+\mathscr{L}^{1 / 3} y^{2 / 3} B\left(\frac{1}{2},\left|F-L^{\prime} G-L F G\right|^{4 / 3}\right)+\eta^{2} r
$$

$\ll \mathscr{L}^{1 / 3} y^{4 / 3} B\left(\theta,\left(L^{\prime} / L+F\right)^{4}\right)^{1 / 3} B\left(\theta,(L G-1)^{2}\right)^{2 / 3}$

$$
+\mathscr{L}^{1 / 3} y^{2 / 3} B\left(\frac{1}{2}, 1+|L|^{4}+\left|L^{\prime}\right|^{4}\right)^{1 / 3} B\left(\frac{1}{2}, 1+|F|^{4}\right)^{1 / 3} B\left(\frac{1}{2}, 1+|G|^{4}\right)^{1 / 3} .
$$


Thus, by Lemmas 2.1 and 2.2,

$$
\begin{aligned}
\Sigma_{3} & \ll y^{4 / 3+\varepsilon}\left(1+\eta^{2} r y^{-1 / 2}\right)^{2 / 3}+y^{2 / 3+\varepsilon}\left(\eta^{2} r\right)^{2 / 3}\left(y+\eta^{2} r\right)^{1 / 3} \\
& \ll y^{4 / 3+\varepsilon}+y^{1+\varepsilon}\left(\eta^{2} r\right)^{2 / 3}+y^{2 / 3+\varepsilon} \eta^{2} r .
\end{aligned}
$$

Hence, by (4.3) and (4.10),

$$
\Sigma_{1}(\eta) \ll y^{1+\varepsilon} \eta^{1 / 2} q^{1 / 2} r^{1 / 4}+y^{3 / 4+\varepsilon} \eta^{3 / 2} q^{1 / 2} r^{3 / 4}+y^{1 / 2+\varepsilon} \eta^{2} q^{1 / 2} r .
$$

Therefore, by (4.2),

$$
\sum_{m \leqslant \xi} \Psi(m q) \ll y^{3 \varepsilon}\left(y q^{-1 / 4}+y^{3 / 4} \xi^{1 / 2} q^{1 / 4}+y^{1 / 2} \xi q^{1 / 2}+\xi^{3 / 2} q^{1 / 2}\right) .
$$

This implies Lemma 3.1 .

\section{Another analogue of the Bombieri-Vinogradov theorem}

Let

$$
\begin{gathered}
y_{1}=\lambda_{0}+\lambda_{1} y \\
\mathscr{N}=\mathscr{N}(X)=\left\{p_{1} p_{2} p_{3} p_{4} p_{5}: X \leqslant p_{1} \leqslant y_{1}^{1 / 5}, p_{1} \leqslant p_{2} \leqslant\left(\frac{y_{1}}{p_{1}}\right)^{1 / 4},\right. \\
\left.p_{2} \leqslant p_{3} \leqslant\left(\frac{y_{1}}{p_{1} p_{2}}\right)^{1 / 3}, p_{3} \leqslant p_{4} \leqslant\left(\frac{y_{1}}{p_{1} p_{2} p_{3}}\right)^{1 / 2}, X \leqslant p_{5} \leqslant \frac{y_{1}}{p_{1} p_{2} p_{3} p_{4}}\right\}, \\
\mathscr{D}^{*}=\mathscr{D}^{*}(X)=\left\{n: n \leqslant y,\left\|\lambda_{0}+\lambda_{1} n\right\| \leqslant \frac{1}{2} z, \llbracket \lambda_{0}+\lambda_{1} n \rrbracket \in \mathscr{N}\right\}, \\
\vartheta(k, j, h)=\sum_{\substack{n \in \mathcal{N} \\
n=j(\bmod k)}} 1,
\end{gathered}
$$

and

Further write

$$
x_{1}=\lambda_{1}{ }^{-1} z|\mathscr{N}| .
$$

$$
R_{r}^{*}=\left|\mathscr{D}_{r}^{*}\right|-x_{1} r^{-1} .
$$

Theorem 5.1. Suppose that $0<\delta \leqslant \frac{1}{50}$ and $y^{1 / 16-\delta / 3}<X<y^{1 / 6}$. Then on the hypothesis of Theorem 3.1,

$$
\sum_{r \leqslant y^{1 / 4-\delta}}\left|R_{r}^{*}\right| \ll y^{1-\delta / 4}
$$

Our procedure is similar to that of $\S 33$ and 4 , but is sufficiently different because of the nature of $\mathscr{D}^{*}$ for it to be necessary to give the details. As in $\S 3$ the proof depends on a lemma.

Lemma 5.1. Let

and

$$
\Theta(u, r, h)=\sum_{\substack{m \leqslant u \\(m, r)=1}}\left(\vartheta(r, m, h)-\frac{|\mathcal{N}|}{\varphi(r)}\right)
$$

$$
\Theta(r)=\max |\Theta(u, r, h)| \text {, }
$$


where the maximum is taken over all $u, h$ with $u \leqslant q\left(2+\left|\lambda_{0}\right|\right)$ and $(h, r)=1$. Then

$$
\sum_{m \leqslant y^{1 / 4-\delta}} \Theta(m a) \ll y^{1-2 \delta / 7}
$$

To deduce the theorem from the lemma we argue as follows. By (5.2) and (5.3),

$$
\begin{aligned}
\left|\mathscr{D}_{r}^{*}\right| & =\sum_{\substack{m, n, n \in \mathscr{N}, r|m\\
| \lambda_{0}+\lambda_{1} m-n \mid \leqslant \leq z}} 1+O(1) \\
& =\sum_{\substack{n \in \mathcal{S},\left\|\left(n-\lambda_{0}\right) / \lambda_{1}\right\| \leqslant z / 2 \lambda_{1} \\
r \mid \mathbb{(}\left(n-\lambda_{0}\right) / \lambda_{1} \rrbracket}} 1+O(1) .
\end{aligned}
$$

Write $-\lambda_{0} / \lambda_{1}=\left(b+\theta_{2}\right) / a$ with $\left|\theta_{2}\right| \leqslant 1$ and note that

$$
1 / \lambda_{1}=q / a+\theta_{3} /\left(\lambda_{1} a q\right)
$$

with $\left|\theta_{3}\right| \leqslant 1$. Thus by repeating the argument of $\S 3$ we have

Let

$$
\begin{aligned}
\left|\mathscr{D}_{r}^{*}\right| & =\sum_{|j| \leqslant \varepsilon a / 2 \lambda_{1}} \vartheta(a r, j-b, q)+O\left(\frac{y(q, r)}{q^{\delta} r}+q^{1-\delta}\right) \\
& =\sum_{h,|b+h| \leqslant z a / 2 \lambda_{1}} \vartheta(a r, h, q)+O\left(\frac{y(q, r)}{q^{\delta} r}+q^{1-\delta}\right) .
\end{aligned}
$$

Then, by (5.11),

By (5.4),

$$
\left|\mathscr{D}_{r}^{*}\right|=\sum_{j,|b / g+j| \leqslant \varepsilon a / 2 \lambda_{1} g} \vartheta\left(a \frac{r}{g}, j, \frac{q}{g}\right)+O\left(\frac{y g}{q^{\delta} r}+q^{1-\delta}\right) .
$$

$$
\begin{aligned}
\sum_{\substack{j,(j, a r / g)>1 \\
|b / g+j| \leqslant \varepsilon a / 2 \lambda_{1} g}} \vartheta\left(a \frac{r}{g}, j, \frac{q}{g}\right) & \ll \sum_{j,|b / g+j| \leqslant z a / 2 \lambda_{1} g} \sum_{\substack{p m \in \mathcal{N}, p \mid(j, a r / g) \\
(q / g) p m \equiv j(\bmod a r / g)}} 1 \\
& \ll \sum_{\substack{p \mid a r / g \\
p \geqslant X}} \sum_{\substack{h \\
|b /(g p)+h| \leqslant \varepsilon a / 2 \lambda_{1} g p(q / g) m \equiv h(\bmod a r / g p)}} 1 .
\end{aligned}
$$

This is easily seen to be

$$
\begin{aligned}
& \ll \sum_{\substack{p \mid a r / g \\
p \geqslant X}}\left(\frac{z a}{g p}+1\right)\left(\frac{y_{1} g}{a r}+1\right) \\
& \ll z y \mathscr{L} X^{-1} r^{-1}+y \mathscr{L} g q^{-1} r^{-1}+z q \mathscr{L} X^{-1}+\mathscr{L} .
\end{aligned}
$$

Hence, by (5.13),

$$
\left|\mathscr{D}_{r}^{*}\right|=\sum_{\substack{j,|b / g+j| \leqslant z a / 2 \lambda_{1} g \\(j, a r / g)=1}} \vartheta\left(a \frac{r}{g}, j, \frac{q}{g}\right)+O\left(\frac{y \mathscr{L} g}{q^{\delta} r}+q^{1-\delta}\right) .
$$


Thus, by (5.6), (5.5), and (3.12),

$$
R_{r}^{*} \ll\left|\sum_{\substack{j,(j, a r / g)=1 \\|b / g+j| \leqslant z a / 2 \lambda_{1} g}}\left(\vartheta\left(a \frac{r}{g}, j, \frac{q}{g}\right)-\frac{|\mathscr{N}|}{\varphi(a r / g)}\right)\right|+y \mathscr{L} g q^{-\delta} r^{-1} .
$$

We note that $\left(z a / 2 \lambda_{1}\right)+|b| \leqslant q+\left(a / \lambda_{1}\right)\left|\lambda_{0}\right| \leqslant q\left(2+\left|\lambda_{0}\right|\right)$. Theorem 5.1 now follows from (5.14) and Lemma 5.1.

\section{The proof of Lemma 5.1}

By (5.2),

$$
\sum_{\substack{n \in \mathcal{N} \\(n, r)>1}} 1 \ll \sum_{\substack{p \mid r \\ p \geqslant X}} \sum_{m \leqslant y_{1} / p} 1 \ll y \mathscr{L} X^{-1}
$$

Hence, by (5.8) and (5.4),

$$
\Theta(u, r, h)=\frac{1}{\varphi(r)} \sum_{\chi \neq \text { Хomod } r}\left(\sum_{m \leqslant u} \bar{\chi}(m)\right) \chi(h) \vartheta(\chi)+O\left(y u \mathscr{L} X^{-1} \varphi(r)^{-1}\right),
$$

where $\vartheta(\chi)=\Sigma_{n \in \mathscr{N}} \chi(n)$. Thus, by the analogue of (4.1), and (5.9),

$$
\Theta(m a) \ll y q \mathscr{L} X^{-1} \varphi(m a)^{-1}+\frac{d(m a)}{\varphi(m a)} \sum_{\chi \neq \chi_{0 \text { mod } m a}} \max _{u \leqslant q\left(2+\left|\lambda_{0}\right|\right)}\left|\sum_{j \leqslant u} \chi^{*}(j) \vartheta_{m a}\left(\chi^{*}\right)\right|,
$$

where

$$
\vartheta_{r}(\chi)=\sum_{n \in \mathscr{N},(r, n)=1} \chi(n)
$$

Hence, if $\xi \leqslant y^{1 / 4-\delta}$,

$$
\begin{aligned}
\sum_{m \leqslant \xi} \Theta(m a) & \ll y^{1-\delta}+y^{8} q^{-1} \sum_{r \mid a} \max _{k \leqslant y} \sum_{r-1<n \leqslant \xi} n^{-1} \sum_{\chi_{\bmod n r}} \max _{u \leqslant q\left(2+\left|\lambda_{0}\right|\right)}\left|\sum_{j \leqslant u} \chi(j) \vartheta_{k}(\chi)\right| \\
& \ll y^{1-\delta}+y^{8} q^{-1} \sum_{r \mid a} \max _{k \leqslant y}\left(\xi^{-1} \Sigma_{4}(\xi, k)+\int_{1}^{\xi} \Sigma_{4}(\eta, k) \eta^{-2} d \eta\right),
\end{aligned}
$$

where

$$
\Sigma_{4}(\eta, k)=\sum_{r-1<n \leqslant \eta} \sum_{\chi \bmod n r}^{*} \max _{u \leqslant q\left(2+\left|\lambda_{0}\right|\right)}\left|\sum_{j \leqslant u} \chi(j) \vartheta_{k}(\chi)\right|
$$

Let

$$
\Sigma_{5}=\sum_{r-1<n \leqslant \eta} \sum_{\chi \bmod n r}^{*}\left|\vartheta_{k}(\chi)\right|^{4 / 3}
$$

Then, by (6.3), (4.4), and Hölder's inequality,

Let

$$
\Sigma_{4}(\eta, k) \leqslant \Sigma_{2}^{1 / 4} \Sigma_{5}^{3 / 4} \text {. }
$$

$$
\begin{gathered}
\mathscr{M}=\left\{m: m=p_{1} p_{2} p_{3} p_{4}, X \leqslant p_{1} \leqslant y_{1}^{1 / 5}, p_{1} \leqslant p_{2} \leqslant\left(y_{1} / p_{1}\right)^{1 / 4}\right. \\
\left.p_{2} \leqslant p_{3} \leqslant\left(y_{1} /\left(p_{1} p_{2}\right)\right)^{1 / 3}, p_{3} \leqslant p_{4} \leqslant\left(y_{1} /\left(p_{1} p_{2} p_{3}\right)\right)^{1 / 2}\right\} \\
\mathscr{M}_{1}=\left\{m: m \in \mathscr{M}, m \leqslant y^{1 / 2}\right\}
\end{gathered}
$$

and

$$
\mathscr{M}_{2}=\left\{m: m \in \mathscr{M}, m>y^{1 / 2}\right\} \text {. }
$$


Then, by (6.1) and (5.2),

where

$$
\left|\partial_{k}(\chi)\right|^{4 / 3} \ll\left|\vartheta_{1, k}(\chi)\right|^{4 / 3}+\left|\vartheta_{2, k}(\chi)\right|^{4 / 3},
$$

Let

$$
\vartheta_{j, k}(\chi)=\sum_{\substack{m, p, m \in \mathscr{M}_{j} \\ X \leq p \leq \mathcal{M}_{1} / m \\(m p, k)=1}} \chi(m p) .
$$

$$
\begin{aligned}
& D_{j}(s, \chi)=\sum_{\substack{m \in \mathscr{M}_{j} \\
(m, k)=1}} \chi(m) m^{-8}, \\
& E_{1}(s, \chi)=\sum_{\substack{p \geqslant \max \left(\eta^{2} r, X\right) \\
p \nmid k}} \chi(p) p^{-s} \quad(\sigma>1), \\
& E_{2}(s, \chi)=\sum_{\substack{p \geqslant \max \left(\eta 1^{1 / 2}, X\right) \\
p \nmid k}} \chi(p) p^{-s} \quad(\sigma>1) \text {, } \\
& H_{1}(s, \chi)=\sum_{\substack{x \leqslant p<\eta^{2} \\
p \nmid k}} \chi(p) p^{-8},
\end{aligned}
$$

and

$$
H_{2}(s, \chi)=\sum_{\substack{X \leq p<\eta r^{1 / 2} \\ p \nmid k}} \chi(p) p^{-s}
$$

Further, let

$$
y_{0}=\frac{1}{2}+\max _{m \leqslant y_{1}} m \text {. }
$$

Then for a non-principal character $\chi$ to a modulus not exceeding $y$, we have, by Lemma $2.6,(4.6),(4.7)$, and (6.10),

$$
2 \pi i \vartheta_{j, k}(\chi)=\int_{\theta-i T}^{\theta+i T}\left(D_{j} E_{j}\right)(s, \chi) \frac{y_{0}^{s}}{s} d s+\int_{1 / 2-i T}^{1 / 2+i T}\left(D_{j} H_{j}\right)(s, \chi) \frac{y_{0}^{8}}{s} d s+O(1) .
$$

Hence, by Hölder's inequality,

$$
\begin{aligned}
\left|\vartheta_{j, k}(\chi)\right|^{4 / 3} \ll y^{4 / 3} \mathscr{L}^{1 / 3} \int_{-T}^{T}\left|\left(D_{j} E_{j}\right)(\theta+i t, \chi)\right|^{4 / 3} \frac{d t}{1+|t|} \\
\\
+y^{2 / 3} \mathscr{L}^{1 / 3} \int_{-T}^{T}\left|\left(D_{j} H_{j}\right)\left(\frac{1}{2}+i t, \chi\right)\right|^{4 / 3} \frac{d t}{1+|t|}+1 .
\end{aligned}
$$

Thus, by Hölder's inequality,

$$
\begin{aligned}
& \sum_{r-1<n \leqslant \eta} \sum_{\chi \bmod n r}^{*}\left|\vartheta_{1, k}(\chi)\right|^{4 / 3} \\
& \ll y^{4 / 3} \mathscr{L}^{1 / 3}\left(B\left(\theta, D_{1}^{4}\right)\right)^{1 / 3}\left(B\left(\theta, E_{1}^{2}\right)\right)^{2 / 3} \\
& \quad+y^{2 / 3} \mathscr{L}^{1 / 3}\left(B\left(\frac{1}{2}, D_{1}^{4}\right)\right)^{1 / 3}\left(B\left(\frac{1}{2}, H_{1}^{2}\right)\right)^{2 / 3}+\eta^{2} r
\end{aligned}
$$

Therefore, by Lemma 2.2, (6.6), (6.7), (6.11), (6.12), and (6.14),

$$
\begin{aligned}
\sum_{r-1<n \leqslant \eta} & \sum_{\chi \text { mod }}^{*}\left|\vartheta_{1, k}(\chi)\right|^{4 / 3} \\
& \ll y^{4 / 3+\varepsilon}\left(1+\eta^{2} r X^{-8}\right)^{1 / 3}+y^{2 / 3+\varepsilon}\left(y+\eta^{2} r\right)^{1 / 3}\left(\eta^{2} r\right)^{2 / 3}+\eta^{2} r \\
& \ll g^{4 / 3+\varepsilon}+y^{7 / 6+\delta}\left(\eta^{2} r\right)^{1 / 3}+y^{1+8}\left(\eta^{2} r\right)^{2 / 3}+y^{2 / 3+8} \eta^{2} r .
\end{aligned}
$$


Similarly

$$
\begin{aligned}
& \sum_{r-1<n \leqslant \eta} \sum_{\chi \bmod r r}^{*}\left|\vartheta_{2, k}(\chi)\right|^{4 / 3} \\
& \quad \ll y^{4 / 3} \mathscr{L}^{1 / 3}\left(B\left(\theta, D_{2}{ }^{2}\right)\right)^{2 / 3}\left(B\left(\theta, E_{2}{ }^{4}\right)\right)^{1 / 3} \\
& \quad+y^{2 / 3} \mathscr{L}^{1 / 3}\left(B\left(\frac{1}{2}, D_{2}^{2}\right)\right)^{2 / 3}\left(B\left(\frac{1}{2}, H_{2}^{4}\right)\right)^{1 / 3}+\eta^{2} r \\
& \quad \ll y^{4 / 3+\varepsilon}\left(1+\eta^{2} r y^{-1 / 2}\right)^{2 / 3}+y^{2 / 3+\varepsilon}\left(y^{4 / 5}+\eta^{2} r\right)^{2 / 3}\left(\eta^{2} r\right)^{1 / 3}+\eta^{2} r \\
& \quad \ll y^{4 / 3+\varepsilon}+y^{6 / 5+\varepsilon}\left(\eta^{2} r\right)^{1 / 3}+y^{1+\varepsilon}\left(\eta^{2} r\right)^{2 / 3}+y^{2 / 3+\varepsilon} \eta^{2} r .
\end{aligned}
$$

Hence, by (6.4) and (6.9),

$$
\Sigma_{5} \ll y^{4 / 3+\varepsilon}+y^{6 / 5+\varepsilon}\left(\eta^{2} r\right)^{1 / 3}+y^{1+\varepsilon}\left(\eta^{2} r\right)^{2 / 3}+y^{2 / 3+\varepsilon} \eta^{2} r .
$$

Therefore, by (4.10) and (6.5),

$$
\begin{aligned}
& \Sigma_{4}(\eta, k) \\
& \quad \ll y^{1+\varepsilon} \eta^{1 / 2} q^{1 / 2} r^{1 / 4}+y^{9 / 10+8} \eta q^{1 / 2} r^{1 / 2}+y^{3 / 4+\varepsilon} \eta^{3 / 2} q^{1 / 2} r^{3 / 4}+y^{1 / 2+\varepsilon} \eta^{2} q^{1 / 2} r .
\end{aligned}
$$

Thus, by (6.2),

$$
\sum_{m \leqslant \xi} \Theta(m a) \ll y^{38}\left(y q^{-1 / 4}+y^{9 / 10}+y^{3 / 4}\left(\xi^{2} q\right)^{1 / 4}+y^{1 / 2}\left(\xi^{2} q\right)^{1 / 2}\right) .
$$

Lemma 5.1 now follows easily.

7. The proof of the main theorem

Let

and

$$
\begin{gathered}
\delta=10^{-5}, \\
\tau=\frac{1}{6} \delta, \\
\alpha=\frac{1}{4}-\delta, \\
\left|\lambda_{1}-a / q\right| \leqslant q^{-2}, \quad q>q_{0}\left(\lambda_{0}, \lambda_{1}\right), \quad(a, q)=1, \\
y=q^{2 /(1+\delta),} \\
z=y^{-\delta / 5} \\
x=z \pi(y), \\
u=4(1+\delta) /(1-4 \delta),
\end{gathered}
$$

Let

$$
\lambda=1 /(6-u-\delta) \text {. }
$$

$$
V=\sum_{\substack{b \in \mathscr{O} \\\left(b, P\left(x^{\alpha / 4}\right)\right)=1}}^{\prime \prime}\left(1-\sum_{\substack{\alpha / 4 \\ x^{\prime} \leqslant p<x^{1 / u}}} \lambda(1-(u(\log p) / \log x))\right),
$$

where the "indicates that the summation is restricted to those elements of $\mathscr{D}$ which do not have repeated prime factors. By $(3.1),\left|\mathscr{D}_{r}\right| \ll 1+y / r$. 
Hence, by Lemma 2.3 and Theorem 3.1,

$$
\begin{aligned}
V & \geqslant \frac{2 x}{\alpha \log x} \\
& \times\left(\log 3-\lambda(1+\delta) \log 4+\lambda(1+\delta) \log (1+\delta)+\lambda \delta \log (3 / \delta)+O\left((\log x)^{-1 / 15}\right)\right) .
\end{aligned}
$$

By (7.8) and (7.9), $0<\lambda-\frac{1}{2}<6 \delta$. Hence

$$
\begin{aligned}
\lambda(1+\delta) \log 4 & -\lambda(1+\delta) \log (1+\delta)-\lambda \delta \log (3 / \delta) \\
& <\left(\frac{1}{2}+6 \delta\right)(\log 4+\delta \log 4-13 \delta)<\log 2+4 \delta .
\end{aligned}
$$

Hence, by (7.11) and (7.3),

$$
V>\frac{8 \log \frac{3}{2}-32 \delta}{1-4 \delta} \frac{x}{\log x} .
$$

Thus, by (7.1),

$$
V>3 \cdot 243 x / \log x .
$$

Consider the definition of $V,(7.10)$. The weight in the sum satisfies

$$
1-\sum_{\substack{\alpha / / 4 \\ p \mid b<x^{1 / u}}} \lambda\left(1-u \frac{\log p}{\log x}\right) \leqslant 1-\lambda\left(\Omega(b)-u \frac{\log |b|}{\log x}\right) .
$$

Hence, by (3.1), (7.5), (7.7), (7.9), and (7.1),

$$
\begin{aligned}
1-\sum_{\substack{x^{\alpha / 4} \leq p<x^{1 / 4} \\
p \mid b}} \lambda\left(1-u \frac{\log p}{\log x}\right) & \leqslant \lambda\left(6-\Omega(b)+u \frac{\log \left(\lambda_{0}+\lambda_{1} y+\frac{1}{2} z\right)}{\log x}-u-\delta\right) \\
& <\lambda(6-\Omega(b)) .
\end{aligned}
$$

Thus the weight is negative if $\Omega(b)>5$ and is at most $\lambda$ if $\Omega(b)=5$. Moreover, every element of $\mathscr{D}$ for which there is a positive contribution to $V$ has no prime factor less than $x^{\alpha / 4}$, and is squarefree. It therefore suffices to show that the contribution to $V$ from those elements of $\mathscr{D}$ having exactly five prime factors is at most $3.041 x / \log x$. By (3.1), (5.2), and (5.3) it is thus enough to show that

$$
\lambda S\left(\mathscr{D}^{*}\left(x^{\alpha / 4}\right), y^{1 / 4-\delta}\right)<3.041 x / \log x .
$$

By Lemma 2.4, Theorem 5.1, and (7.3),

$$
S\left(\mathscr{D}^{*}\left(x^{\alpha / 4}\right), y^{1 / 4-\delta}\right)<\left(\frac{8}{1-4 \delta}+\delta\right) \frac{x_{1}}{\log x_{1}} .
$$

Hence, by (7.9), (7.8), (5.5), (5.2), (5.1) and (7.6),

$$
\lambda S\left(\mathscr{D}^{*}\left(x^{\alpha / 4}\right), y^{1 / 4-\delta}\right)<(4+100 \delta) x_{1} / \log x .
$$


We now proceed to estimate $x_{1}$. By (5.2), (5.1), and the prime number theorem,

$$
\begin{aligned}
\left|\mathscr{N}\left(x^{\alpha / 4}\right)\right|< & (1+\varepsilon) y_{1} \int_{y_{1} 1_{1 / 16-\delta / s}^{1 / 5}}^{y_{1 / 3}} \frac{d u_{1}}{u_{1} \log u_{1}} \int_{u_{1}}^{\left(y_{1} / u_{1}\right)^{1 / 4}} \frac{d u_{2}}{u_{2} \log u_{2}} \int_{u_{2}}^{\left(y_{1} / u_{1} u_{2}\right)^{1 / 3}} \frac{d u_{3}}{u_{3} \log u_{3}} \\
& \times \int_{u_{3}}^{\left(y_{1} / u_{1} u_{3} u_{3}\right)^{1 / 2}} \frac{d u_{4}}{u_{4}\left(\log u_{4}\right) \log \left(y_{1} / u_{1} u_{2} u_{3} u_{4}\right)} \\
< & \lambda_{1}(1+\delta) \pi(y)(I+500 \delta),
\end{aligned}
$$

where

$$
I=\int_{1 / 16}^{1 / 5} \frac{d v_{1}}{v_{1}} \int_{v_{1}}^{\left(1-v_{1}\right) / 4} \frac{d v_{2}}{v_{2}} \int_{v_{2}}^{\left(1-v_{1}-v_{2}\right) / 3} \frac{d v_{3}}{v_{3}} \int_{v_{3}}^{\left(1-v_{1}-v_{2}-v_{3}\right) / 2} \frac{d v_{4}}{v_{4}\left(1-v_{1}-v_{2}-v_{3}-v_{4}\right)}
$$

The substitution $1-v_{1}-\ldots-v_{j-1}=v_{j} u_{j}$ gives

$$
\begin{aligned}
I & =\int_{5}^{16} \frac{d u_{1}}{u_{1}-1} \int_{4}^{u_{1}-1} \frac{d u_{2}}{u_{2}-1} \int_{3}^{u_{2}-1} \frac{d u_{3}}{u_{3}-1} \int_{2}^{u_{3}-1} \frac{d u_{4}}{u_{4}-1} \\
& =\int_{4}^{15} \frac{d v_{1}}{v_{1}} \int_{4}^{v_{1}} \frac{d v_{2}}{v_{2}-1} \int_{4}^{v_{2}} \frac{\log \left(v_{3}-3\right)}{v_{3}-2} d v_{3} \\
& =\int_{4}^{15} \frac{\log \left(v_{3}-3\right)}{v_{3}-2} d v_{3} \int_{v_{3}}^{15} \frac{d v_{2}}{v_{2}-1} \int_{v_{2}}^{15} \frac{d v_{1}}{v_{1}} \\
& \leqslant \int_{4}^{15} \frac{u \log (u-3)}{(u-1)(u-2)} d u \int_{u}^{15} \frac{1}{v} \log \frac{15}{v} d v \\
& =\frac{1}{2} \int_{4}^{15} \frac{u \log (u-3)}{(u-1)(u-2)}\left(\log \frac{15}{u}\right)^{2} d u .
\end{aligned}
$$

We compute an upper bound for $I$ as follows. Let

$$
J(j)=\frac{(j+1)(j-2)}{j(j-1)}\left(\log \frac{15}{j}\right)^{2}\left(\log ^{2}(j-2)-\log ^{2}(j-3)\right) .
$$

Then

$$
\begin{aligned}
I & \leqslant \frac{1}{2} \sum_{j=4}^{14} \int_{j}^{j+1} \frac{u(u-3)}{(u-1)(u-2)}\left(\log \frac{15}{u}\right)^{2} \frac{\log (u-3)}{u-3} d u \\
& \leqslant \frac{1}{4} \sum_{j=4}^{14} J(j) .
\end{aligned}
$$

In the following table, $\bar{J}(j)$ denotes a number such that $\bar{J}(j) \geqslant J(j)$.

$\begin{array}{rrrrrr}j & \bar{J}(j) & j & \bar{J}(j) & j & \bar{J}(j) \\ 4 & 0.70 & 8 & 0.24 & 12 & 0.03 \\ 5 & 0.79 & 9 & 0.15 & 13 & 0.01 \\ 6 & 0.57 & 10 & 0.09 & 14 & 0.01 \\ 7 & 0.38 & 11 & 0.05 & & \end{array}$


(This table was computed with the use of a table of five-figure natural logarithms. It was then checked on an HP65.) Hence, by (7.17), $4 I \leqslant 3.02$. Therefore, by (7.15), (5.5), (7.14), and (7.7), we have (7.13).

\section{REFERENCES}

1. JING-RUN Chen, 'On the representation of a larger even integer as the sum of a prime and the product of at most two primes', Sci. Sinica 16 (1973) 157-76.

2. H. DavenPort, Multiplicative number theory (Markham, Chicago, 1967).

3. - and H. Harberstak, 'The values of a trigonometrical polynomial at well spaced points', Mathematika 13 (1966) 9I-96.

4. - and K. F. Rотн, 'The solubility of certain diophantine inequalities', ibid. 2 (1955) 81-96.

5. P. X. Gallagher, 'The large sieve', ibid. 14 (1967) 14-20.

6a. H. Halberstam and H.-E. Richert, Sieve methods (Academic Press, London, 1974).

6. 'A large sieve density estimate near $\sigma=1$ ', Invent. Math. 11 (1970) 329-39.

7. L. K. HUa, Additive theory of prime numbers, Amer. Math. Soc. Transl. 13 (Providence, R.I., 1965).

8. H. L. Montgomery, Topics in multiplicative number theory, Lecture Notes in Mathematics 227 (Springer-Verlag, Berlin, 1973).

9. K. Prachar, Primzahlverteilung (Springer-Verlag, Berlin, 1957).

10. H.-E. RICHent, 'Selberg's sieve with weights', Mathematika 16 (1969) 1-22.

11. P. M. Ross, 'On Chen's theorem that each large even number has the form $p_{1}+p_{3}$ or $p_{1}+p_{2} p_{3}$ ', J. London Math. Soc., (2) 10 (1975) 500-6.

12. E. C. TItchmarSh, The theory of the Riemann zeta-function (Clarendon Press, Oxford, 1951).

13. R. C. Vafghan, 'Diophantine approximation by prime numbers, I', Proc. London Math. Soc. (3) 28 (1974) 373-84.

14. 'Diophantine approximation by prime numbers, II', ibid. (3) 28 (1974) 385-401.

15. - 'Mean value theorems in prime number theory', J. London Math. Soc. (2) 10 (1975) 153-62.

\section{Department of Mathematics}

Imperial College

London S.W.7

and

The University of Michigan

Ann Arbor, Michigan 48104 